\title{
Schweigen und Schreiben. Albert Camus' algerisches Dilemma
}

\author{
Von Cornelia RuHe (Mannheim)
}

\begin{abstract}
:
Bereits mit seinen journalistischen Schriften hatte Albert Camus sich bemüht, ein Bild von Algerien und seinen Bewohnern zu zeichnen, wie es seiner Sicht als Algerienfranzose entsprach. Mit seinem letzten Roman Le premier homme versucht er, seiner Herkunft aus einem städtischen Milieu armer Algerienfranzosen ein literarisches Denkmal zu setzen und ihnen damit Eingang zu verschaffen in die französische Literatur und in das kollektive Gedächtnis der Franzosen. Zugleich stellt der Roman auch seine lang erwartete Stellungnahme zum Algerienkrieg dar, die nicht öffentlich, sondern literarisch erfolgen sollte. Es ist die Aufgabe der vorliegenden Untersuchung, den erst 1994 postum erschienenen Roman literarisch in dem intertextuellen Umfeld zu situieren und zu analysieren, in dem er entstanden ist und gelesen werden wollte: Le premier homme ist, so die These, als Einreihung Camus' in eine neue, dezidiert maghrebinische Schreibtradition zu interpretieren.

C'est déjà avec ses écrits journalistiques qu'Albert Camus essaya d'esquisser une image de l'Algérie et des Algériens qui correspondait à sa vue en tant que Français d'Algérie. Avec son dernier roman Le premier homme, paru de manière posthume en 1994, Camus tente d'ériger un monument littéraire en l'honneur de son milieu d'origine, celui des algérois pauvres pour leur permettre de faire leur entrée dans la littérature française et de s'inscrire dans la mémoire collective des Français. Le roman constitue en même temps sa prise de position à propos de la guerre d'Algérie qui ne sera pas, comme l'on s'y attendait, publique, mais littéraire. La présente étude situera et analysera le roman dans le contexte intertextuel dans lequel il a été conçu: Le premier homme sera interprété comme l'inscription de Camus dans une nouvelle tradition d'écriture résolument maghrébine.
\end{abstract}

Keywords:

Albert Camus, Albert Memmi, maghrebinische Autobiographie, Algerienkrieg, Intertextualität

Albert Camus, Albert Memmi, autobiographie maghrébine, guerre d'Algérie, intertextualité 
Ce qu'on ne peut pas dire, il ne faut surtout pas le taire, mais il faut l'écrire.

(Jacques Derrida ${ }^{1}$ )

C'est parce qu'il avait pris quelque distance avec la question algérienne que Camus l'a comprise mieux que quiconque.

(Boualem Sansal ${ }^{2}$ )

\section{Entre-deux}

Ein kleiner Junge geht mit seiner Großmutter ins Kino. Es handelt sich, wie zur damaligen Zeit üblich, um einen Stummfilm. Die Großmutter ist Analphabetin und verlangt energisch, der Junge möge ihr die Zwischentitel laut vorlesen. Gegen diese Störung der Vorstellung protestieren die anderen Zuschauer. Da er sich nicht in der Lage sieht, sowohl den Ansprüchen seiner Großmutter als auch den Bitten des Publikums gleichermaßen nachzukommen, verstummt der Junge schließlich völlig.

Zwischen zwei Parteien, die gleichermaßen legitime Ansprüche an ihn stellen, denen zu gehorchen einerseits die gesellschaftlichen Umgangsformen und andererseits der Respekt einem älteren Familienmitglied gegenüber gebieten, wählt Jacques Cormery - so der Name des Jungen - den dritten Weg des Schweigens. Als Ausweg aus dem Dilemma entschließt sich das Kind nicht für eine der beiden Seiten, sondern für eine eigene Position. Er ordnet sich nicht den Erwartungen der Anderen unter. Diese unabhängige Haltung wird sich auch sein Autor Albert Camus sein Leben lang bewahren.

Als kleiner Junge im Kino sieht Jacques sich inmitten der «exigences de la grand-mère et $[\mathrm{d}]$ es remontrances de plus en plus irritées de ses voisins» ${ }^{3}$ außerstande, einen Mittelweg zu finden, der den Ansprüchen aller zu genügen vermag - aus dem einfachen Grund, dass es einen solchen Mittelweg im Falle der skizzierten Situation nicht gibt. Es wird nicht das letzte Mal sein, dass auch der Autor dieses autobiographischen Romans sich in einer solch dilemmatischen Lage wiederfindet.

Mit dem Ausbruch des Algerienkrieges beginnt für Albert Camus eine Zeit, die ihn als Algerienfranzosen nicht nur in beständiger Sorge um Familie und Freunde in Algier sein lässt. Seine wachsende Berühmtheit, die in der Verleihung des Nobelpreises für Literatur gipfelt, macht ihn zu einem der exponiertesten Intellektuellen seines Landes, ja zu einer moralischen Instanz. Seine Herkunft scheint ihn zudem dazu zu prädestinieren, im Algerienkonflikt eine

\footnotetext{
${ }^{1}$ Jacques Derrida: La Carte postale - de Socrate à Frend et au-delà. Paris: Flammarion 1980, 209.

2 Boualem Sansal: «Préface». In: Jacques Ferrandez/Albert Camus: L’hôte. Paris: Gallimard 2009, 5.

3 Albert Camus: Le premier homme. Paris: Gallimard 1994, $92 \mathrm{ff}$.
} 
klare Haltung zu haben, an der weniger informierte Mitmenschen sich orientieren könnten ${ }^{4}$.

Camus, der bereits lange vor dem eigentlichen Beginn der 〈guerre sans nom〉 auf die Mißstände infolge des französischen Kolonialismus in Algerien eindringlich hingewiesen hatte ${ }^{5}$, wird von beiden Konfliktparteien als Gewährsmann reklamiert: Diejenigen Algerienfranzosen, die sich für eine Fortsetzung des kolonialen status quo einsetzen, verstehen ihn als einen der ihren, dessen Fürsprache für ihre Sache - die der 〈Algérie française - sie als evident voraussetzen. Die politische Linke in Frankreich hingegen, die sich zunehmend auf die Seite der algerischen Unabhängigkeitsbewegung stellt, versteht ihn als ehemaligen Kommunisten und nun Sozialisten als selbstverständlichen Parteigänger ihrer Position. Beide Seiten versuchen ihn für sich zu gewinnen. Er, der doch die Bedingungen vor Ort kennt, soll die wenigen Bilder sowie die offiziellen und journalistischen Verlautbarungen zu den <événements kommentieren, soll sie für ein Publikum von «métropolitains`, die mit der Realität der Kolonie nicht vertraut sind, übersetzen und verständlich machen. Bei seinen Kommentaren soll er allerdings von den von den jeweiligen ideologischen Positionen vorgeformten Mustern nicht abweichen. Da Camus die Erfahrung gemacht hatte, dass seine unparteiischen Stellungnahmen ihm den Unmut beider Seiten zuzogen ${ }^{6}$, entschloss er sich in dem Moment zu schweigen, in dem

${ }^{4}$ Gegen diese Vereinnahmung von Autoren protestiert Albert Camus bezeichnenderweise 1958, im Vorwort zu seinen Chroniques algériennes: «Mon opinion, d'ailleurs, est qu'on attend trop d'un écrivain en ces matières. Même, et peut-être surtout, lorsque sa naissance et son cœur le vouent au destin d'une terre comme l'Algérie, il est vain de le croire détenteur d'une vérité révélée» (Camus (1958): Chroniques algériennes. In: Ders.: Essais. Paris: Gallimard 2000, 887-1018, hier 900). Die moralische Verantwortung des Autors jenseits der politischen Lager steht vielmehr für ihn im Vordergrund: «il n'y a pas pour l'artiste de bourreaux privilégiés. C'est pourquoi la beauté, même aujourd'hui, surtout aujourd'hui, ne peut servir aucun parti [...]. Le seul artiste engagé est celui qui, sans rien refuser du combat, refuse du moins de rejoindre les armées régulières, je veux dire le franc-tireur» (Camus: «Conférence du 14 décembre 1957». In: Camus 2000, 10771096, hier 1092). Siehe hierzu auch die Rede Camus' mit dem Titel «Ce que je dois à l'Espagne» von 1958, in der das Thema wieder aufgegriffen wird (in: Camus 2000, 19051908).

5 Siehe hierzu vor allem die Reportage Misère de la Kabylie von 1939, in der Camus eindringlich die Gleichstellung der «indigènes» mit den Franzosen einfordert: «si l'on veut vraiment d'une assimilation et que ce peuple si digne soit français, il ne faut pas commencer par le séparer des Français. Si je l'ai bien compris, c'est tout ce qu'il demande. [...] Mais c'est à nous de faire tomber les murs qui nous séparent.» Camus: Misère de la Kabylie. In: Camus 2000 (Anm. 4), 905-938, hier 923.

${ }^{6}$ Siehe hierzu auch James Le Sueur: Uncivil War. Intellectuals and Identity Politics during the Decolonization of Algeria. Lincoln/London: University of Nebraska Press 2005, 100: "Camus's decision to remain silent came only after he participated in public debates, and the public (French and Algerian Muslims alike) found his stance favoring Franco-Arab reconciliation not only unacceptable but also anachronistic». 
Reden von ihm vielleicht am meisten verlangt war: in den Jahren nach der Verleihung des Nobelpreises?

Ebenso wie sein Protagonist in der von ihm in seinem posthum erschienenen autobiographischen Text Le premier homme skizzierten Szene im Kino befindet Camus sich zwischen zwei Parteien mit unvereinbaren Interessen, die ihm seine Position jeweils vorschreiben wollen. Keine der beiden angebotenen Positionen entspricht jedoch seiner Situation. Nimmt er zum Konflikt Stellung, so tut er dies aus einer anderen, dritten Position heraus, die in dem in zwei Lager zerfallenen Frankreich nicht vorgesehen ist. Zwischen der antikolonialen und tendenziell kommunistischen Linken und der prokolonialen Rechten, die die terroristischen Gewalttaten der jeweils Anderen verurteilen und sie zugleich nutzen, um ihrerseits den Einsatz von Terror und Gewalt zu rechtfertigen, scheint es keine dritte Position zu geben.

Seit dem Tod von Camus 1960 versuchen zahlreiche Kritiker, ihn anhand seiner journalistischen wie literarischen Texte der einen oder anderen Seite zuzuschlagen ${ }^{8}$. Allzu häufig geht es dabei weniger um Camus als Autor als vielmehr um die Frage, ob seine Positionen aus historischer Perspektive als richtig oder falsch, naiv oder kritisch einzustufen seien. Selbst die ansonsten interessante und differenzierte Studie von David Carroll befindet sich auf der Suche nach dem, was in Camus' Texten «wrong» oder «right» gewesen sei ${ }^{9}$. Hinter diesen Positionierungen verbirgt sich nach wie vor die Polarisierung, die der Streit zwischen Sartre und Camus nicht nur in der damaligen französischen Gesellschaft auslöste. Was ursprünglich ein Streit zwischen zwei Freunden über politisch-moralische Gesinnungen war, wächst sich im vorherrschenden gesellschaftlichen Klima zu einem Urteil nicht nur über Camus, den philosophischen und politischen Denker, aus, sondern wird zu einem Verdikt auch über seine im eigentlichen Sinne literarischen Texte.

7 Robert Zaretsky datiert den Beginn dieses Schweigens bereits auf das Jahr 1957 (Robert Zaretsky: Albert Camus. Elements of a Life. Ithaca/London: Cornell University Press 2010, 4). Im Umfeld der Verleihung des Nobelpreises kommt es allerdings zu einer von der französischen Presse viel und missverständlich kommentierten Aussage ( JJe crois à la justice, mais je défendrai ma mère avant la justice»). In den folgenden zwei Jahren bis zu seinem Tod wird Camus sich nicht mehr öffentlich zum Algerienkrieg äußern.

${ }^{8}$ Für eine Diskussion der gegensätzlichen Positionen in der Kritik siehe David Carroll: Albert Camus the Algerian. Colonialism, Terrorism, Justice. New York: Columbia University Press 2007, 11ff. Für Conor Cruise O’Brien und mehr noch für Edward Saïd etwa ist er zwar ein «moral man in an immoral situation» (Edward Saï: «Camus and the French Imperial Experience». In: Ders.: Culture and Imperialism. London: Vintage 1993, 204-224, hier 210), er rechtfertige aber in seinen literarischen Texten die französische Herrschaft über Algerien.

9 Carroll 2007 (Anm. 8), xiii. 
Camus selbst, dessen Positionen in einigen Fällen, wie Robert Zaretsky anmerkt, "ha[ve] since become orthodoxy» ${ }^{10}$, hat sich solch einfachen dichotomischen Setzungen stets verweigert. Camus' Ambivalenz, sein Zaudern, seine Weigerung, Ideologien und (Vor)Verurteilungen blind zu folgen, waren in den hochgradig ideologisch und politisch aufgeladenen Kontexten, in denen er sich bewegte, nicht gefragt. ${ }^{11}$ Heute mag diese Haltung immer noch unbequem sein, nach dem Zusammenbruch der Großideologien und Totalitarismen, denen Camus stets skeptisch gegenüberstand, ist sie umso aktueller.

Bei einem so vielschichtigen Autor wie Camus ist es naheliegend, nicht nur seine journalistischen Texte oder öffentlichen Äußerungen, sondern auch die Romane, Erzählungen, Theaterstücke und Essays danach zu befragen, welche Position eingenommen wird. Dabei sollte es aber weniger - wie bei Camus nur allzu häufig geschehen - darum gehen, diese Texte als Abbilder der Realität zu lesen und ihm so z.B. das Fehlen klar konturierter berberischer oder arabischer Protagonisten zum Vorwurf zu machen oder gar nach ‘wahr〉 oder 〈falsch〉 zu suchen, sondern vielmehr darum, die im eigentlichen Sinne literarischen Qualitäten seiner Texte, die vielleicht auf den ersten Blick weniger evidenten literarischen Filiationen, in die Camus sich einschreibt, zu erkennen und vor dem Hintergrund der politischen Situation lesbar zu machen. Dies soll im Folgenden anhand einer Analyse des posthum erschienenen autobiographischen Romans Le premier homme geschehen. Mit diesem Roman, so soll gezeigt werden, gedachte Camus, sein Schweigen zu brechen und in literarischer Form Stellung dazu zu nehmen, wie er sich ein zukünftiges Algerien vorstellte.

\section{Koloniale Dichotomien}

Wenn Camus über Algerien spricht und schreibt, so tut er dies, je nach der Perspektive des Kritikers, aus einer kolonialen oder antikolonialen Haltung her-

10 Zaretsky 2010 (Anm. 7), 6.

${ }^{11}$ Christiane Chaulet Achour arbeitet z.B. heraus, dass die Position Camus' zum Algerienkonflikt, die aus seinen ebenso journalistischen wie essayistischen Texten hervorgehe, im Gegensatz zu der anderer Algerienfranzosen wie Jean Sénac als konservativ zu bezeichnen sei: «droit à la justice pour les 〈Arabes〉, réforme du système colonial mais transformation qui ne peut se faire que dans un lien étroit avec la France; il n'y a jamais acceptation d'une Nation algérienne indépendante où les Français d'Algérie qui voudraient continuer à vivre dans leur pays le pourraient, en acceptant un statut de minoritaires» (Christiane Chaulet Achour: «Albert Camus face à la question algérienne». In: o.N.: 50 ans après les accords d'Évian. Entre mémoire et histoire - regards croisés, regards apaisés. Paris 2013 [im Druck]). An anderer Stelle arbeitet sie allerdings selbst mit Bezug auf Octavio Paz - heraus, dass das, was als Mangel an kritischem Potential aufgefasst werden könnte, sich der «complexité coloniale» verdanke (Christiane Chaulet Achour: «La 〈Méditerranée»: un détournement du colonial? Albert Camus et d'autres écrivains d’Algérie». In: Mustapha Trabelsi (Hg.): Albert Camus et la Méditerranée. Paris 2014 [im Druck]). 
aus. Der Kontext, in dem er sich bewegt, war allerdings nicht nur politisch, sondern auch diskursiv kolonial geprägt: Frankreich schafft in einem Land, in dem die Willkür seiner kolonialen Expansion Berber, Araber, Tuareg, Juden, Türken, Spanier, Franzosen, Malteser und viele Andere unter einem Dach zusammengefasst hat, zwei klar distinkte Parteien, die den wesentlich vielschichtigeren Bedingungen vor Ort nicht zu entsprechen vermögen. Im Algerienkrieg wird diese zunächst administrative Aufteilung dazu genutzt, beide Seiten einander in vermeintlich eindeutiger und unausweichlicher Weise gegenüberzustellen. Dieser klaren Front, die jede Argumentation auf unhintergehbare Dichotomien zwischen Kolonisierenden und Kolonisierten zurückführt, fallen in der öffentlichen Wahrnehmung und letztlich auch in der historischen Realität insbesondere die jüdische algerische Bevölkerung sowie die pieds noirs zum Opfer.

Rechtlich war diese Spaltung des Landes zum Zeitpunkt des Ausbruchs des Unabhängigkeitskrieges längst vollzogen: Das Dekret Crémieux von 1870 erklärt die algerischen Juden zu Franzosen. Die muslimische Bevölkerung verbleibt im Status der <indigènes`, den zuvor auch die jüdische Bevölkerung innehatte und für den eingeschränkte Rechte gelten. Der Historiker Benjamin Stora bezeichnet die Konsequenzen dieses Dekrets als «premier exil»" ${ }^{12}$, als das erste Exil der algerischen Juden. Die Veränderung der Rechtslage bewirkt, dass die algerischen Juden, deren Familien gemeinsam mit den Muslimen teilweise seit Jahrhunderten auf dem Territorium des heutigen Algeriens lebten, plötzlich den Status von Europäern und Franzosen erhielten, der einer historischen Grundlage entbehrte ${ }^{13}$. Juden und Muslime, die sich unabhängig von ihrer Religionszugehörigkeit vor der Ankunft der Franzosen 1830 gleichermaßen

12 Benjamin Stora: Les trois exils. Juifs d'Algérie. Paris: Stock 2006, 13.

13 Gleichwohl muss festgehalten werden, dass sich die jüdischen Gemeinden in Frankreich ab 1830 um die jüdische Bevölkerung Algeriens bemühen und so etwa bereits ab 1832 beginnen, gemischte Schulen auf algerischem Boden zu gründen, in denen der Unterricht auf französisch erfolgt. Ungeachtet ihrer historischen Provenienz werden die algerischen Juden ab diesem Zeitpunkt gezielt französisiert. Für den Historiker Benjamin Stora ist die auch administrative Loslösung der algerischen Juden von ihren maghrebinischen Wurzeln aber erst mit dem Dekret Crémieux vollzogen: «Cette rupture [gemeint ist das Dekret] d'une vieille tradition liée à la terre d'Algérie s'est traduite en premier lieu par une perte progressive de la langue arabe. Ils apprennent le français et l'histoire de France en tenant graduellement à l'écart leur héritage hébraïque et arabe, et sont entraînés sur la voie de l'assimilation républicaine» (Stora 2006 [Anm. 12], 13). Dieser Prozess hatte, so führt Stora aus, mit Beginn der französischen Kolonisierung Algeriens strategischen Charakter: «Dès 1831, la monarchie française s'est donc cmployée à supprimer l'autonomie de la nation juive en posant, mesure après mesure, les actes qui conduiront à la naturalisation collective des juifs d'Algérie du 24 octobre 1870, connue sous le nom de décret Crémieux. Cette politique découle des premières observations sur cette population et de l'évaluation de son rôle éventuel dans le processus de colonisation de l'Algérie.» Stora 2006 (Anm. 12), 36-37. 
einheimisch gefühlt hatten, was sich seit 1830 in der gemeinsamen Zugehörigkeit zur Gruppe der «indigènes gezeigt hatte, werden nun anhand einer von der Kolonialverwaltung gezogenen konfessionellen Linie gespalten ${ }^{14}$. Stora betont, dass diese bewusste Zerstörung der bisherigen gesellschaftlichen Ordnung eine neue Qualität der kolonialen Landnahme darstellt: «La colonisation française a ainsi créé dans l'espace algérien un modèle d'occupation complètement nouveau qui a fracturé les relations intercommunautaires déjà fortement fissurées par le déclin turc.» ${ }^{15}$ Die Bevölkerung Algeriens wird durch dieses Gesetz faktisch in drei Gruppen aufgeteilt: die der Franzosen mit vollen staatsbürgerlichen Rechten, die der indigènes> sowie die der in Algerien lebenden Ausländer, also Spanier, Maltesen etc. ${ }^{16}$

Die so entstandenen zwei Gruppen, aus denen im Unabhängigkeitskrieg schließlich die zwei Fronten konstruiert werden, sind, wie ihre Genese deutlich macht, allerdings weder ein Spiegel der vorgefundenen Realität noch sind sie in sich homogen: Die Gruppe der so genannten indigènes> lässt sich in Araber, Berber und Tuareg unterteilen, deren Verhältnis zum französischen Kolonialstaat sich je unterschiedlich gestaltet, die im allgemeinen Sprachgebrauch der Einfachheit halber aber unter «les arabes` subsumiert werden. Die Gruppe der zum Teil bereits seit der ersten Hälfte des 19. Jahrhunderts auf algerischem Boden lebenden (Algerien)Franzosen wiederum erweitert sich 1870 um die jüdische Bevölkerung Algeriens, die unter Vichy vorübergehend ihre Rechte als Staatsbürger verliert. Zu dieser Gruppe gehören ab 1889 auch die zu unterschiedlichen Zeitpunkten nach Algerien gekommenen Spanier, Italiener und anderen in Algerien lebenden Europäer, die nun naturalisiert werden. Sie alle werden, wie nach der Unabhängigkeit Algeriens besonders deutlich wird, von den Festlandfranzosen als <anders> wahrgenommen und sind sich selbst auch ihrer Eigenheiten bewußt. Im Verwaltungs- und Bildungsbereich der Kolonie arbeiten darüber hinaus zahlreiche Festlandsfranzosen, die nicht dort verwurzelt sind und daher kein starkes affektives Verhältnis zum Land haben.

Ein Effekt der binären Gegenüberstellung von «Français` und «indigènes〉 ist, dass der Gruppe der «Français', der ja auch die pieds noirs und die algerischen Juden angehören, von der algerischen Unabhängigkeitsbewegung faktisch ihr Aufenthaltsrecht in Algerien aberkannt wird: Sie sind eben nicht <indigènes». Camus setzt sich dagegen vehement zur Wehr: «Les Français d'Algérie sont, eux aussi, et aux sens fort du terme, des indigènes.» ${ }^{17}$

${ }^{14}$ Es muss allerdings betont werden, dass unter ottomanischer Herrschaft keine Gleichheit zwischen den Angehörigen der verschiedenen Religionsgemeinschaften geherrscht hatte, vielmehr unterstanden Juden und Christen dem Dekret dhimmi, das ihnen strenge Auflagen erteilt (siehe hierzu Stora 2006 [Anm. 12], 31).

15 Stora 2006 (Anm. 12), 42.

16 Dank eines Gesetzes von 1889 wurden dann auch sie naturalisiert und mehrten die Gruppe der Franzosen.

17 Albert Camus: «Algérie 1958». In: Camus 2000 (Anm. 4), 1011-1015, hier 1013. 
Die hybride Gesellschaft, die die postkolonialen Kulturwissenschaften meist erst im späten 20. Jahrhundert vorzufinden meinen, existierte folglich mindestens in dieser Kolonie vor der französischen Eroberung, bevor die Kolonialherren sie diskursiv einebneten und somit dichotomisch wurde, was weit vielschichtiger gewesen war ${ }^{18}$. Den Blick dafür zu öffnen, dass diese auf Opposition und Konflikt ausgerichteten Binarismen von den Kolonialmächten gezielt geschaffen wurden, um komplexere und damit auch kompliziertere Realitäten zu verbergen und zu vereinfachen, ist die Aufgabe einer neueren Generation von postkolonialen Studien, so formulieren es auch Frederick Cooper und Ann Laura Stoler:

It does us no service to reify a colonial moment of binary oppositions so that we can enjoy the postcolonial confidence that our world today is infinitely more complicated, more fragmented and more blurred. [...] We need to think through not only a colonial history that appears as Manichaean but a historiography that has invested in that myth as well. ${ }^{19}$

Die politisch-gesellschaftlichen Dichotomien, die für den Algerienkrieg so wichtig werden sollten, sind folglich keine Abbildung einer (prä)kolonialen Lebensrealität, sondern vielmehr diskursive Setzungen, die durch die aus ihnen abgeleitete ungleiche Verteilung der Privilegien auf die Lebensrealität zurückwirken. ${ }^{20}$

Die Untergliederung der kolonialen Gesellschaft in nur zwei Gruppen hat vorgeblich administrative Gründe. Die sich aus ihr ergebende drastische Komplexitätsreduktion hat erhebliche Rückwirkungen auf das Zusammenleben der Gruppen $^{21}$, aber auch - und das wird während des Algerienkrieges eine wich-

${ }^{18}$ Die gezielte Politik der Arabisierung Algeriens nach Erlangung der Unabhängigkeit ist, ähnlich der dichotomen Unterteilung zu kolonialen Zeiten, als eine Strategie der politisch motivierten Identitätsbildung zu verstehen, die die Pluralität des Landes und seiner Bevölkerung negiert (etwa in der Unterdrückung der Berbersprachen).

${ }_{19}$ Frederick Cooper/Ann Laura Stoler: «Between Metropole and Colony. Rethinking a Research Agenda». In: Dies. (Hg.): Tensions of Empire. Colonial Cultures in a Bourgeois World. Berkeley: University of California Press 1997, 1-56, hier 9.

${ }^{20}$ Cooper und Stoler stellen fest: «Colonial dichotomies of ruler and ruled, white and black, colonizer and colonized only reflected part of the reality in which people lived. As we have stressed, these dichotomies took hard work to sustain, were precariously secured, and were repeatedly subverted. [...] ambivalence was part of colonial encounters for colonizer and colonized.» Cooper/Stoler 1997 [Anm. 19], 34.

${ }^{21}$ Benjamin Stora hat dies exemplarisch an den Auswirkungen der Kolonialpolitik auf das Zusammenleben von jüdischen und muslimischen Algeriern nachgewiesen: «La naturalisation collective des juifs d'Algérie a créé un bouleversement considérable dans l'univers de leur communauté qui vivait alors, sinon en symbiose, du moins avec la population musulmane. Après cette séparation, du jour au lendemain, ils changent de camp et se solidarisent avec l'envahisseur. Recensés, inscrits à l'état civil, ils apprennent à lire et à écrire le français, abandonnent les petits métiers traditionnels pour embrasser des professions nouvelles sans rien renier de leurs coutumes religieuses ou culinaires.» Stora 2006 (Anm. 12), 54. 
tige Rolle spielen - für die Außenwahrnehmung auf dem mit den Gegebenheiten vor Ort nicht vertrauten französischen Festland oder im Ausland.22

Sie dienen den Zielen der französischen Kolonialherrschaft, indem sie Konfliktparteien schaffen, deren Solidaritäten in entscheidenden Momenten einfach abzurufen sind. Konsequenzen hat diese willentlich hergestellte Opposition zweier allenfalls theoretisch homogener Gruppen für alle über den Algerienkrieg möglichen Aussagen: Wenn andere Solidaritäten als die mit Franzosen oder aufständischen «indigènes> nicht vorgesehen sind, dann werden sie nicht gehört, sie sind gleichsam nicht existent bzw. werden, wo sie zum Beispiel aufgrund der Prominenz des Redners nicht ignoriert werden können, für falsch oder naiv erklärt.

Camus' eigene Position, die eines Algerienfranzosen, der sich keinen Fortbestand des status quo wünschte, sondern vielmehr ein französisches Algerien, in dem die muslimische Bevölkerung gleiche Rechte wie die Franzosen erhielte, eine Position also, die Forderungen beider Seiten vereinte, fand in der französischen Gesellschaft keine Resonanz. ${ }^{23}$

\section{Historische Kontextualisierung}

Die Algerienfranzosen, zu denen auch Camus selbst gehört, werden insbesondere von den antikolonial engagierten französischen Intellektuellen mit Beginn der Unabhängigkeitsbewegung als homogene Gruppe konstruiert: Sie, die Franzosen in Algerien, verfügten über umfangreiche Ländereien, beuteten das Land aus und bereicherten sich auf Kosten der indigènes`. Jeder Algerienfranzose, so wird insinuiert, entspräche diesem sorgfältig konstruierten Feindbild und sei damit als Vertreter des Kolonialismus zu bekämpfen.

${ }^{22}$ Im Verlauf des Krieges werden schließlich auch die algerischen indigènes mit vollen Bürgerrechten versehen, allerdings zu einem Zeitpunkt, zu dem dieser Schritt an der Wahrnehmung insbesondere der Festlandfranzosen bereits nichts mehr ändern konnte: «As nationalist violence escalated and as the French government increased its propaganda efforts, Algerian Muslims - who were made French citizens during the war - became (for many moderate and right-wing French intellectuals) less and less ‘French,, less and less ‘Western', and more and more islamic〉, more and more «Muslim», or simply more and more 〈Other〉.» Le Sueur 2005 (Anm. 6), 13.

${ }^{23}$ Mit Michel Foucault kann man hier das Wirken der diskursiven Disziplin erkennen, vor allem aber auch den doktrinären Charakter der von den beiden Konfliktparteien vertretenen Positionen: «die Zugehörigkeit zu einer Doktrin geht sowohl die Aussage wie das sprechende Subjekt an - und zwar beide in Wechselwirkung. Durch die Aussage und von der Aussage her stellt sie das sprechende Subjekt in Frage, wie die Ausschließungsprozeduren und die Verwerfungsmechanismen beweisen, die einsetzen, wenn ein sprechendes Subjekt eine oder mehrere unzulässige Aussagen gemacht hat; Häresie und Orthodoxie sind nicht fanatische Übertreibungen der Doktrinmechanismen: sie gehören wesenhaft zu ihnen» (Michel Foucault: Die Ordnung des Diskurses. Frankfurt/Main: Fischer Wissenschaft 1991, 29). Camus befindet sich mit seinen Aussagen für beide Seiten im Bereich der Häresie. 
Camus hatte dieser "condamnation en bloc sur les Français d'Algérie», dieser «opinion métropolitaine, qui ne se lasse pas de les haï» ${ }^{24}$ stets widersprochen. Das homogene Bild der Algerienfranzosen korrigiert er mit Rekurs auf die Geschichte seiner Familie, eine Haltung, die ihm von den Kritikern des Kolonialismus als naiv vorgeworfen worden ist:

Je résume ici l'histoire des hommes de ma famille qui, de surcroit, étant pauvres et sans haine, n'ont jamais exploité ni opprimé personne. Mais les trois quarts des Français d'Algérie leur ressemblent et, à condition qu'on les fournisse de raisons plutôt que d'insultes, seront prêts à admettre la nécessité d'un ordre plus juste et plus libre. Il y a eu sans doute des exploiteurs en Algérie, mais plutôt moins qu'en métropole et le premier bénéficiaire du système colonial est la nation française tout entière. $^{25}$

Bereits mit seinen journalistischen Schriften hatte Camus sich bemüht, ein Bild von Algerien und seinen Bewohnern zu zeichnen, das realistisch war und die Wissenslücken der Festlandfranzosen über Land und Leute füllte. Mit seinem letzten Roman nun versucht er, seiner Herkunft aus einem städtischen Milieu armer Algerienfranzosen ein literarisches Denkmal zu setzen und ihnen damit Eingang zu verschaffen nicht nur in die Literatur, sondern auf diesem Wege auch in das kollektive Gedächtnis der Franzosen. Zugleich stellt der Roman auch seine lang erwartete Stellungnahme zum Algerienkrieg dar, die eben nicht, wie es von ihm erwartet wird, öffentlich, sondern literarisch erfolgen sollte.

Camus' Roman erscheint aufgrund seines Unfalltods erst 1994, in einer Zeit, in der der Algerienkrieg seit langem beendet, die postkoloniale Kritik auf ihrem Höhepunkt und der Kommunismus, mit dem Camus sich intensiv auseinandergesetzt hatte, zusammengebrochen war. Der Text, mit dem Camus das Schweigen über Algerien zu brechen gedachte, den er als eine ebenso literarische wie literaturpolitische Stellungnahme in diesem Konflikt konzipierte, erscheint so erst zu einem Zeitpunkt, als die Unabhängigkeit Algeriens bereits seit über 30 Jahren eine historische Tatsache ist. Es ist die Aufgabe der vorliegenden Untersuchung, den Roman literarisch in dem intertextuellen Umfeld $\mathrm{zu}$ situieren und $\mathrm{zu}$ analysieren, in dem er entstanden ist und gelesen werden wollte. ${ }^{26}$

24 Camus 1958 (Anm. 4), 897.

25 Camus 1958 (Anm. 4), 897. Siehe hierzu auch Benjamin Stora: Le livre, mémoire de l'Histoire. Réflexions sur le livre et la guerre d'Algérie. Paris: Les éditions du préau des collines 2005, 183: «Ils [les pieds noirs] n'ont pas un niveau de vie supérieur à leurs compatriotes métropolitains, et ne comprennent pas le procès qu'ils leur font à propos de leurs prétendues richesses (ils sont accusés de ‘faire suer le burnous`)».

${ }^{26}$ Seine Tochter Catherine Camus betont im Kommentar zur amerikanischen Ausgabe, die Publikation so lange hinausgezögert zu haben, um das Andenken an ihren Vater zu schützen: «In denouncing totalitarianism, and in advocating a multicultural Algeria where both communities would enjoy the same rights, Camus antagonized both the 
Selbst für einen Autor, der bislang so zurückhaltend mit Informationen über seine Familie umgegangen war wie Albert Camus, erscheint es naheliegend, das Projekt eines Romans zum Algerienkrieg im Modus des autobiographischen Romans anzugehen. Die persönliche Fundierung verspricht, dem Roman nicht nur eine große Leserschaft, sondern auch die gewünschte Aufmerksamkeit von Seiten der Kritik zu verschaffen.

Meine These ist allerdings, dass die Wahl des Genres sich überwiegend anderen Gründen verdankte: Die Richtung des literarischen Einflusses, die von der Kritik (zum Teil bis heute) in gut kolonialer Manier als einseitig, nämlich von Europa nach Afrika, verstanden wird, kehrt Camus um. Um die Algerienfranzosen literarisch zu verewigen, wählt er zwar das traditionelle Genre des autobiographischen Romans, allerdings nicht in seiner althergebrachten Form, sondern in der, in der es damals dank seiner maghrebinischen Kollegen erneut Popularität erlangt und dabei darüber hinaus neue Impulse erfahren hat, die er sich nun seinerseits aneignet.

In den 50er Jahren erscheinen in französischen Verlagen mehrere autobiographische Texte, deren Autoren aus den damaligen französischen Kolonien stammen. Mouloud Feraoun ${ }^{27}$, Camara Laye ${ }^{28}$, Albert Memmi ${ }^{29}$, Driss Chraibi $^{30}$ u.a. publizieren zwischen 1950 und 1954 ihre jeweils ersten Romane, die auf eigene Erfahrungen zurückgehen. Sie werden nicht nur als meist antikoloniale Gründungstexte sich herausbildender (National)Literaturen betrachtet, sondern stellen auch auf dem Gebiet der Autobiographie Neuerungen dar. Sie werden von der französischen Literaturkritik allerdings nicht als solche wahrgenommen:

Sous leurs plumes [celles des autobiographes post-coloniaux], l'autobiographie s'est transformée de l'intérieur, en relation étroite avec des modalités de vie qu'on ne pouvait exprimer par des formes traditionnelles. Puisque la critique littéraire métropolitaine a toujours considéré les auteurs venus des pays colonisés comme

right and the left. At the time of his death he was very much isolated and subject to attacks from all sides designed to destroy the man and the artist so that his ideas would have no impact [...]. In these circumstances, to have published an unfinished manuscript [...] might well have given ammunition to those who were saying Camus was through as a writer.» Catherine Camus in Albert Camus: The First Man. New York: Vintage 1995, vi.

${ }^{27}$ Mouloud Feraoun: Le fils du pauvre. Le Puy 1950. Hier wird nach der Ausgabe Paris: Seuil 1954 zitiert, während die in der Ausgabe Paris 1954 weggelassenen Kapitel über die Zeit an der École normale de la Bouzaréa nach folgender Ausgabe zitiert werden: «Fouroulou Menrad». In: Mouloud Feraoun: L'anniversaire. Paris: Seuil 1972, 103141.

28 Camara Laye: L'enfant noir. Paris: Plon 1953.

29 Albert Memmi: La statue de sel. Paris: Corréa 1953. Hier wird nach der Ausgabe Paris: Gallimard 1966 zitiert.

30 Driss Chraibi: Le passé simple. Paris: Denoël 1954. Der Roman wird später bei Gallimard aufgelegt. 
les élèves dociles des maîtres européens de la parole et ne s'attendait pas à ce qu'ils puissent innover, elle ne s'en aperçut pas. Elle ne soupçonna même pas que des révolutions pussent se préparer en marge de leur propre aire culturelle et en dehors du canon littéraire établi. ${ }^{31}$

Die Romane der vier Autoren sind zwar bei der Kritik erfolgreich, werden aber in der Tradition vorgängiger französischer autobiographischer Texte gesehen ${ }^{32}$, ohne das innovative Potential zu erkennen oder zu würdigen. Vielmehr werden sie in einer Übertragung des Verhältnisses von Kolonisator und Kolonisiertem als von der französischen Literatur beeinflusst betrachtet, da man davon ausgeht, dass wahre Neuerungen auch auf dem Gebiet der Literatur nicht von den Kolonien ausgehen können. Man konzentriert sich auf die Kontinuität, anstatt die Innovationen zu sehen, durch die die jungen Autoren eine traditionell gefestigte europäische Gattung verändert und bereichert haben ${ }^{33}$.

Debra Kelly geht in ihrer Untersuchung Autobiography and Independence (in der sie unter anderem Texte von Memmi und Feraoun analysiert) von folgenden Besonderheiten (post)kolonialer autobiographischer Texte aus:

Many critics analysing North African literature in French [...] will maintain that the collective voice is more important than the individual, that every $\langle\mathrm{I}\rangle$ masks a (we), that the writer speaks on behalf of a community that has often been deprived of a voice, due to colonialism, or due to slavery in the case of America. In the work of writers active during the 1950 s, those most directly linked to independence movements in North Africa, there is certainly the will to srepresent), in both meanings of the word (to portray and to speak on behalf of), the experience of their colonised peoples, while simultaneously forging a process through which an independent subjectivity could come into being after being denied by the colonising forces. In so doing, these writers contributed to the analysis of the effects of colonisation and of resistance to that oppression on the identity of the individual and of the nation. ${ }^{34}$

31 Doris Ruhe: «Le scorpion en phénix. L'écriture autobiographique d'Albert Memmi». In: Alfred Hornung/Ernstpeter Ruhe (Hrsg.): Postcolonialisme E Autobiographie: Albert Memmi, Assia Djebar, Daniel Maximin. Amsterdam/Atlanta: Rodopi 1998, 53-67, hier 54.

32 So weist auch Guillemette Tison einzig auf die - vorhandenen, aber wenig frappierenden - Parallelen zu Jean-Paul Sartres Autobiographie Les mots hin (Guillemette Tison: «La culture de l'enfant dans Le premier homme». In: Roman 20/50 27 (1999), 3951, hier 50-51.

${ }_{33} \mathrm{Zu}$ dieser gängigen Form der Abweisung fremder> literarischer Innovation siehe auch Cornelia Ruhe: 〈Invasion aus dem Osten>. Die Aneignung russischer Literatur in Frankreich und Spanien (1880-1910). Frankfurt/Main: Vittorio Klostermann Verlag 2012, vor allem $35 \mathrm{ff}$. sowie $96 \mathrm{ff}$.

${ }^{34}$ Debra Kelly: Autobiography and Independence: Selfhood and Creativity in North African Postcolonial Writing in French. Liverpool: Liverpool University Press 2005, 23. Kelly deutet am Ende ihrer Untersuchung an, dass auch Camus' Le premier homme in ihr Korpus hätte aufgenommen werden können (338). Den Tabubruch, einen stets als 
Im Unterschied zu westlich-europäischen Autobiographien, in denen das Individuum und die Individualität eine zentrale Rolle spiele, ginge es hier darum, einem Kollektiv Ausdruck zu verleihen. Der Protagonist im Zentrum stehe also stets für mehr als nur sich selbst, sei als Sprecher einer «community» zu verstehen, der die Stimme bisher versagt blieb. Dabei gehe es in den Texten aus der Zeit der Unabhängigkeitsbewegungen darum, die Erfahrung des kolonisierten Volkes, von dem die Autoren selbst sich aufgrund ihrer Bildungsbiographie entfremdet haben ${ }^{35}$, einer größeren Öffentlichkeit zugänglich zu machen. In diesem Zusammenhang erfolgt, so Kelly, der Versuch der Schaffung einer im doppelten Sinne unabhängigen Identität - hierauf wird noch zurückzukommen sein. ${ }^{36}$

Die Gedanken Kellys lassen sich weiterentwickeln: Die (post)kolonialen Autobiographien insbesondere der 50er Jahre können als Allegorien für im Entstehen begriffene Nationen gelesen werden. Diese These, die Fredric Jameson bereits Mitte der 80er Jahre vertreten hatte ${ }^{37}-$ «All third-world texts are necessarily $[\ldots]$ allegorical, and in a very specific way: they are to be read as what I will call national allegories ${ }^{38}-$, ist mindestens insofern problematisch, als damit im Sinne der oben genannten Abweisung fremden innovativen Poten-

französisch verstandenen Autor auf derselben Ebene wie postkoloniale Autoren zu behandeln, begeht ihre Untersuchung signifikanterweise dennoch nicht.

35 «These writers also have in common the fact that they became, to varying degrees, alienated from their traditional cultural environment and made an intellectual effort in order to cope with that alienation.» Kelly 2005 (Anm. 34), 335. Die Tatsache, dass dieselbe Aussage auf Camus zutrifft, ließe sich als zunächst rein biographische Koinzidenz auch gegen die These der bewussten Einschreibung Camus' in den maghrebinischen literarischen Kontext einwenden: Alle drei Autoren entstammten armen und bildungsfernen> Familien. Der republikanischen Schule und insbesondere dem Einsatz einzelner Lehrer verdankten sie es jeweils, über Stipendien Zugang zur höheren Bildung erhalten und sich so aus ihrem Ursprungskontext gelöst zu haben. Die Parallelität des Erfahrungshorizontes von «indigènes und armen Algerienfranzosen ist andererseits eine Tatsache, die Camus nicht müde wurde zu betonen, um auf diese Weise deutlich zu machen, dass beide Bevölkerungsgruppen sich keineswegs so fremd sind, wie der koloniale, aber auch der antikoloniale Diskurs es behauptete. Diese Parallelität fasst Camus in seinem autobiographischen Roman in Worte und Szenen, die denen seines kabylischen Kollegen Feraoun und insbesondere des Tunesiers Memmi, deren Texte dem seinen vorausgingen, in augenfälliger Weise gleichen.

${ }^{36}$ Hinzufügen könnte man noch, dass zumindest die Texte von Memmi und Feraoun, mit denen der Roman von Camus meiner Ansicht nach im Dialog steht, sich ungeachtet ihrer autobiographischen Bezüge dezidiert nicht der Ich-Form bedienen, sondern ein fiktives Alter Ego in den Vordergrund stellen. Dieses Verfahren kann man als Möglichkeit der Distanzierung von demjenigen kritischen Potential, das die Texte transportieren, verstehen, darin unterscheiden sie sich aber auch von den französischen Autobiographien der damaligen Zeit. Camus übernimmt auch dieses Verfahren.

37 Fredric Jameson: «Third-World Literature in the Era of Multinational Capitalism». In: Social Text 15 (1986), 65-88.

38 Jameson 1986 (Anm. 37), 69. 
tials nicht nur suggeriert wird, alle «Dritte-Welt-Literatur» speise sich einzig und allein aus europäischen Vorbildern, sondern greife vor allem mit der Allegorie auf eine längst überholte literarische Strategie zurück, mit deren Identifikation und Entschlüsselung die meisten Leser des 20. Jahrhunderts nicht mehr vertraut sind. Diese Aussage Jamesons könnte man als subtile Methode werten, die (post)kolonialen Literaturen als vormodern zu stigmatisieren.

Zugleich weist die These Jamesons in eine interessante Richtung: Geht man mit Stephan Leopolds Analyse von Jamesons Text davon aus, dass die Verwendung der Nationalallegorie

gerade davon herrührt, dass sich die nationale Gemeinschaft nicht - oder noch nicht - zu einer imagined community im Sinne Benedict Andersons verfestigt hat, sondern diese imagined community Gegenstand des kollektiven Begehrens ist $^{39}$,

so wäre sie im Falle der hier untersuchten Texte von Memmi und Feraoun der Versuch einer Neuschreibung und Neusituierung der tunesischen bzw. algerischen Nation (auch wenn sie bei Feraoun durchaus konservativ ausfällt). Dass diese im Modus der Allegorie erfolgt, die als überkommenes literarisches Verfahren nicht allen Lesern mehr verständlich ist, ließe sich damit als Methode der Verschleierung einer allzu grundsätzlichen und zugespitzten Kritik an der französischen Kolonialmacht verstehen. Die Romane selbst dienten damit der Reflexion über die zu definierende kollektive und nationale Identität der entstehenden <imagined community. ${ }^{40}$

Camus ist mit den (post)kolonialen Autobiographien Memmis und Feraouns ebenso vertraut wie mit den Autoren selbst. Zum ersten Roman von Albert Memmi verfasst Camus ein Vorwort. Im Falle von Mouloud Feraoun kennt er nicht nur den Text, sondern auch den Autor, mit dem er über mehrere Jahre eine (Brief)Freundschaft pflegt und dem er sich, so Alain Vircondelet, nahe fühlt: «Camus retrouve en lui [Mouloud Feraoun] des échos de sa vie à Belcourt. Ils partagent les mêmes souvenirs d'enfance, le même amour de cette

39 Stephan Leopold: «Problematische Hegemonie, libidinöse Investition. Zur Frage kolonialer Allegoriebildung am Beispiel von Albert Camus (L'Étranger) und Kateb Yacine (Nedjma)». In: Lendemains 130 (2008), 162.

${ }^{40}$ In einer Untersuchung, die die Autorin vorbereitet, wird der Frage nachzugehen sein, wieso die literarische oder filmische Nationalallegorie gerade im Zusammenhang mit dem Schreiben (oder Filmen) über den Spanischen Bürgerkrieg oder den Algerienkrieg vermehrt auftaucht. Ist das im Sinne Jamesons als Zeichen dafür zu werten, dass die imagined communities dieser Länder durch die jeweiligen Kriege erneut brüchig geworden sind und neuerlicher - literarischer und filmischer - Reflexion bedürfen? Die Nationalallegorie wäre dann in mehrfacher Hinsicht - historisch und auf das Genre bezogen als (post)koloniales Erbe zu verstehen. Möglicherweise handelt es sich bei der Allegorie aber auch um eine nicht nur in diesem Zusammenhang bewährte Schreibweise der Bewältigung von (nationalen) Traumata. 
terre pleine de sortilèges.» ${ }^{41}$ Was Vircondelet nur andeutet, soll im Folgenden im Zentrum stehen: Camus, dem die Lebensrealität der jungen maghrebinischen Autoren aufgrund seiner Herkunft vertraut ist, dem das politische Klima diese mehr sentimentale als politische Aneignung aber verweigert, schreibt sich nun nicht mehr durch journalistische Stellungnahmen, sondern durch literarische Affiliation in eine Tradition ein, die ihre Wurzeln im Maghreb und nicht in Europa hat. In dieser zunächst rein literarischen Verankerung in dem Land, das ihm nach eigener Aussage alles bedeutete ${ }^{42}$, steckt, so meine These, auch das politische Erbe Camus'.

\section{Einschreibung in Geschichte und Literatur}

Die Texte von Mouloud Feraoun und Albert Memmi sind ganz im Sinne Debra Kellys von dem Bedürfnis getragen, einer gesellschaftlichen Gruppe, die bisher keinerlei schriftliche Spuren in Geschichte oder Literatur hinterlassen hat, dortselbst Gehör zu verschaffen und ihr damit einen Platz im kollektiven Gedächtnis zu sichern. Mouloud Feraoun, dessen erster Roman in einem herabsetzenden Gestus als «ethnographisch ${ }^{43}$ bezeichnet worden ist, setzt seinem Volk, den Kabylen, ein literarisches Denkmal. Albert Memmi, dessen Protagonist Mordekhaï Alexandre Benillouche die problematische dreifache, jüdische, französische und berberische Herkunft bereits im Namen trägt, führt die berberischen Juden, aus deren Armut und Unbildung der Protagonist sich ebenso wie sein Autor schließlich lösen wird, in die Literatur ein.

Albert Camus macht es sich in seinem Roman zum Ziel, sein «Volk», die Algerienfranzosen, auf Papier zu bannen, in einem für sie historisch prekären Moment: «aujourd'hui où ils risquaient l'anonymat définitif et la perte des seules traces sacrées de leur passage sur cette terre» ${ }^{44}$. Wie den ihm vorgängigen maghrebinischen Autoren geht es auch ihm darum, eine Welt literarisch erstehen zu lassen, die bisher nicht für literaturfähig befunden wurde. Im Unterschied zu Feraoun und Memmi spricht Camus von den pieds noirs bereits als von einer untergehenden Spezies, von einer, die es gilt, durch seinen Roman vor dem endgültigen «anonymat» zu bewahren, in das die Unabhängigkeit Alge-

41 Alain Vircondelet: Albert Camus, fils d'Alger. Paris: Fayard 2010, 347.

42 Besonders emphatisch äußert er diese Verbundenheit in seinem Tagebuch: «Ma terre perdue, je ne vaudrais plus rien.» Camus: Carnets III, mars 1951 - décembre 1959. Paris: Gallimard 1989, 251.

43 Siehe hierzu z.B. Maurice Le Rouzic: «A propos de la dimension autobiographique du Fils du pauvre de Mouloud Feraoun». In: Martine Mathieu (Hg.): Littératures autobiographiques de la francophonie. Paris: L'Harmattan 1996, 45-55; bei Hédi Abdel Jouad werden die Texte Feraouns gar als «ethnozentrisch» betrachtet, Hédi Abdel Jouad: «Ethnography vs. Ethnocentrism in Le fils du pauvre». In: Revue Celfan 1 (1982), 2628.

${ }_{44}$ Camus 1994 (Anm. 3), 181. 
riens sie zurückzuschicken droht - eine Befürchtung, die sich seitdem bewahrheitet hat.

Mit dem Roman verfolgt Camus daher dezidiert politische Ziele: Es gilt nicht, Algerien als eigentlich französisches Territorium zu etablieren, vielmehr geht es ihm um die Betonung der Andersartigkeit nicht nur des Landes, sondern auch seiner Bevölkerung. Camus schreibt, um das Existenzrecht der Algerienfranzosen zu legitimieren, ihr Anrecht auf einen Verbleib in dem einzigen Land, das ihnen Heimat ist. Diese Legitimation bedient sich dabei keiner kolonialpolitischen Argumentation, sondern setzt die Verweildauer im Land als ausschlaggebendes Kriterium an. Gegen die Dichotomisierung der Kolonialverwaltung - français versus indigènes - setzt Camus seine Beschreibung einer modernen convivencia, die nicht frei von prekären Aspekten ist, aber - aus seiner Perspektive - durch die Veränderung einiger Parameter perfektioniert werden könnte. Camus' Text ist damit analog zu den Texten seiner maghrebinischen Vorgänger als Entwurf eines neuen Zusammenlebens jenseits der dichotomischen Hürden des Kolonialstaats zu verstehen.

Die Mischung gerade der armen Bevölkerungsschichten in den Städten ist eine historische Tatsache, der alle drei Autoren Rechnung tragen: Das Zusammenleben in den urbanen Zentren erfolgt zwar in einigen Vierteln wie etwa der Kasbah in Algier nach ethnischen Maßgaben, in anderen hingegen ist es die Klassenzugehörigkeit, die den Ausschlag gibt. Hier verwischen auf vielen Ebenen die Grenzen zwischen den ethnischen und religiösen Gruppen, es entstehen mindestens partiell hybride Identitäten, bei denen Klasse schwerer wiegt als Rasse:

the criteria used to determine who belonged where underscored the permeability of boundaries, opening possibilities for assertion among interstitial groups of 〈mixed-bloods > and <poor whites > as well as those more squarely identified as <the colonized $>{ }^{45}$

Die Verschränkung der überwiegend ethnisch kodierten vertikalen Hierarchie mit der horizontalen Klassenstruktur führt aus der Perspektive der Kolonialmacht zu überraschenden und nicht immer erwünschten Solidaritäten.

Die Protagonisten von Feraoun und Memmi entstammen ebenso wie Jacques Cormery dem, was Camus mit einem gehörigen Schuss Sozialromantik beschreibt als «monde innocent et chaleureux des pauvres, monde refermé sur lui-même comme une île dans la société mais où la misère tient lieu de famille et de solidarité» ${ }^{46}$. Ihre Armut macht insbesondere die städtischen Protagonisten bei Memmi und Camus zu Mitgliedern einer multiethnischen Schicksalsgemeinschaft. Fouroulou Menrad, der anagrammatische Protagonist von Mouloud Feraouns Le fils du pauvre, macht erst auf der École normale de la

\footnotetext{
45 Cooper/Stoler 1997 (Anm. 19), 6.

46 Camus 1994 (Anm. 3), 163.
} 
Bouzaréa die Erfahrung, dass es weniger die ethnische Herkunft als vielmehr die Klasse, der die Familie angehört, ist, die die Freundschaften bedingt:

La race ne réussit pas plus que le clocher à rassembler ses gens. Il reste la condition sociale, la coupe du costume, la situation des parents. [...] Fouroulou, issu d'un milieu fruste, ne peut pas ressembler à tel Français, fils d'un directeur d'école, d'une directrice d'école, ayant un frère professeur. [...] Aucune familiarité. Tel autre, Tlemcenien ou Oranais, fils d'avocat ou d'interprète, bon musulman, citadin pur sang, est aussi étranger à Fouroulou que son camarade français. Il est, certes, beaucoup plus près du fils aîné d'un garçon de café, Espagnol d'origine, ayant cinq ou six enfants et auquel le père de Menrad n'a presque rien à envier. Fouroulou garde aussi le souvenir ému d'un Juif de Médéa, et d'un Arabe de BouSaada avec lesquels il pouvait parler librement de son village et de sa famille parce qu'ils étaient pauvres comme lui. ${ }^{47}$

Die Freundschaften, so wird deutlich, bilden sich aufgrund geteilter biographischer Erfahrungen. Dem Protagonisten von Albert Memmi, der nicht nur zwischen zwei Kulturen, sondern zwischen dreien steht, scheinen sich auf dem lycée hingegen alle schulischen Kontakte zu verschließen: «J'eus des camarades français, tunisiens, italiens, russes, maltais, et juifs aussi, mais d'un milieu si différent du mien qu'ils m'étaient des étrangers. ${ }^{48}$ Weder die Religion noch der gemeinsame berberische Hintergrund können als gemeinsame Grundlage dienen, vielmehr gibt es immer einen Grund, der ihn den Anderen fremd macht:

je suis de culture française, mais Tunisien [...]; je suis Tunisien mais juif, c'està-dire politiquement, socialement exclu, parlant la langue du pays avec un accent particulier, mal accordé passionnellement à ce qui émeut les musulmans; juif mais ayant rompu avec la religion juive et le ghetto, ignorant de la culture juive et détestant la bourgeoisie inauthentique; je suis pauvre enfin et j'ai ardemment désiré en finir avec la pauvreté, mais j'ai refusé de faire ce qu'il fallait. ${ }^{49}$

Während bei Feraoun und Camus dieses Problem überwiegend auf der Ebene von Freundschaften besteht, hat es für den Protagonisten von Memmi Konsequenzen für die identitäre Entwicklung.

Für Jacques Cormery, den Protagonisten von Albert Camus' Roman, stellt sich das Problem zunächst in weniger akuter Weise. Camus hebt hervor, dass es zwar in der «école communale» noch Freundschaften mit «camarades arabes» gegeben, sich das auf dem lycée aber geändert habe: «les lycéens arabes étaient l'exception, et ils étaient toujours des fils de notables fortunés ${ }^{50}$. Sie sind ihm fremd, ebenso wie die «jeunes métropolitains», «que les hasards de la carrière paternelle avaient menés à Alger ${ }^{51}$. Für den Jungen aus armen Verhältnissen

\footnotetext{
${ }^{47}$ Feraoun 1972 (Anm. 27), 111.

48 Memmi 1966 (Anm. 29), 119.

${ }^{49}$ Memmi 1966 (Anm. 29), 364.

50 Camus 1994 (Anm. 3), 187.

51 Camus 1994 (Anm. 3), 190.
} 
sind letztere zwar interessante, aber bislang unbekannte Wesen, da sie im Gegensatz zu ihm über eine (Familien)Geschichte verfügen, die sich in Dokumenten und Fotos, vor allem aber in über Generationen weitergegebenen Erzählungen niederschlägt, die einen Bezug zur in der Schule vermittelten französischen Geschichte aufweisen.

\section{Aufstieg und Spaltung}

Die Spaltung der gemeinsamen Klassenzugehörigkeit, die Einführung ethnischer und religiöser Differenzierungen ist ein Prozess, der in allen drei Romanen mit dem Erkenntniszuwachs der heranwachsenden Protagonisten parallel geführt wird: je älter sie werden, desto bewusster werden sie sich der Unterschiede. Eine zentrale Rolle in dieser Bewusstwerdung spielt die republikanische, in Memmis Fall auch die jüdische Schule, die den Jungen in subtiler Weise diejenigen Vorstellungen vermittelt, die das Fundament für die koloniale Gesellschaft darstellen.

Memmi und Feraoun schreiben ihre Texte aus der Perspektive eines jungen Mannes, der durch die französische Schule einerseits zu Privilegien gelangt ist, die ihm sonst aufgrund seiner Armut nicht zugänglich gewesen wären, die ihn andererseits aber von seinem Ursprungskontext unwiederbringlich getrennt haben. Camus' Protagonist, der dieselbe Karriere durchlaufen hat, ist deutlich älter, seine Perspektive daher zugleich nostalgischer und abgeklärter.

Feraoun spricht von «la plus grande indifférence ${ }^{52}$, die die Eltern seines Protagonisten seinen schulischen Leistungen gegenüber zeigen. Ihr Interesse am akademischen Fortkommen des Jungen ist ein unmittelbareres: Solange er in der Schule ist, isst er zu Hause nicht mit und es bleibt mehr für die Anderen: «Je n'outrepasse pas la vérité en disant que la seule utilité visible de ma scolarisation était mon absence prolongée de la maison qui réduisait la quantité de figues et de couscous que je mangeais. ${ }^{53}$ Die republikanische Schule erweist sich folglich in ganz handfester Weise als Ernährer für die Kinder, denen sie darüber hinaus geistige Nahrung zukommen lässt.

Albert Memmi evoziert ebenfalls die «distance définitive entre la tribu et moi ${ }^{54}$, die die Schule bewirkt. Mit dem Wegfall des Stipendiums durch die jüdische Gemeinde beginnt Alexandre, als «pion» ${ }^{55} \mathrm{zu}$ arbeiten, was ihm Zugang zur Schulkantine der Internatsschüler verschafft. Für ihn ist dieses «régime indigeste et monotone» ${ }^{56}$ eine deutliche Verbesserung gegenüber der Ernährung zu Hause.

${ }^{52}$ Feraoun 1954 (Anm. 27), 62.

53 Feraoun 1954 (Anm. 27), 63.

${ }^{54}$ Memmi 1966 (Anm. 29), 78.

55 Memmi 1966 (Anm. 29), 248.

56 Memmi 1966 (Anm. 29), 249. Allerdings wächst damit auch das schlechte Gewissen darüber, nun wohlgenährter zu sein als seine kleinen Geschwister, die «eux, ne 
Camus' Protagonist ist ebenfalls Stipendiat, «en raison de [sa] «situation de famille» ${ }^{57}$ - die folglich als sehr arm erkannt worden ist. Er hat das Recht, bereits das Frühstück im lycée einzunehmen, gemeinsam mit den Internatsschülern; ein Privileg, das er auf Druck seiner Großmutter auch regelmäßig nutzt ${ }^{58}$.

Dieser evidente Vorteil der Schule ändert gleichwohl nichts daran, dass auch Jacques sich durch sie von seiner Familie entfernt:

Pour la famille de Jacques, le latin par exemple était un mot qui n'avait rigoureusement aucun sens. [...] Dans cette maison où il n'y avait pas de journaux, ni, jusqu'à ce que Jacques en importât, de livres, pas de radio non plus, où il n'y avait que des objets d'utilité immédiate [...], ce que Jacques ramenait du lycée était inassimilable, et le silence grandissait entre sa famille et lui. ${ }^{59}$

Die Möglichkeiten, die die republikanische Schule in gleicher Weise für den armen Algerienfranzosen, Kabylen und tunesischen Juden bereithält, sie sind um einen hohen Preis erkauft: Ohne sie explizit einzufordern, bewirken sie eine - im Falle von Feraouns Protagonisten nur vorübergehende - Herauslösung aus dem Ursprungskontext. Sie entwurzeln die Kinder, ohne ihnen im Gegenzug eine wirkliche neue Heimat jenseits der ihnen zuteil werdenden Bildung anbieten zu können.

Das Paradoxon der Förderung durch die republikanische Schule ist, dass sie ihnen nicht den Schritt über den colonial divide erlaubt, um sie zu <echten> Franzosen mit allen Privilegien zu machen, sondern dass sie sie, um im Bild zu bleiben, in einem Zwischenraum hängen lässt, in dem sie zwar in guter Gesellschaft sind, der allerdings auf diskursiver Ebene im republikanischen Frankreich lange nicht existierte. Vertreten vor allem durch ihre Grundschullehrer nimmt die republikanische Schule, die so entscheidend nicht nur an ihrer Bildung, sondern vor allem auch an der Herausbildung ihrer Identität Anteil hat, sich das Recht heraus, die Kinder kulturell zu entwurzeln, was der Protagonist von Camus retrospektiv positiv bewertet: «[M. Bernard] qui avait jeté Jacques dans le monde, prenant tout seul la responsabilité de le déraciner pour qu'il aille vers de plus grandes découvertes encore.» ${ }^{60}$ Die Entdeckung der Welt geht

mangeaient pas à leur faim. Ils poussaient tout en os, avec de grosses têtes et des jambes noueuses qui n'en finissaient pas.» 250.

57 Camus 1994 (Anm. 3), 202.

58 «Pierre et Jacques avaient obtenu, en raison de leur ssituation de famille, une bourse de demi-pensionnaires. [...] Les classes commençaient à 8 heures ou 9 heures selon les jours, mais le petit déjeuner était servi aux internes à $7 \mathrm{~h} 1 / 4$, et les demipensionnaires y avaient droit. Les familles des deux enfants n'avaient jamais pu imaginer qu'on pût renoncer à un droit quelconque, alors qu'elles en avaient si peu [...].» Camus 1994 (Anm. 3), 202.

59 Camus 1994 (Anm. 3), 186.

60 Camus 1994 (Anm. 3), 149. Im Anhang des Romans (Camus 1994 [Anm. 3], 327) ist der Brief abgedruckt, den Camus nach der Nachricht über den Nobelpreis an seinen 
für die Protagonisten jedoch mit Erfahrungen der Ablehnung einher, vor allem mit einem Gefühl der Fremdheit nicht nur in und gegenüber der anderen Kultur, sondern von nun an auch in der eigenen. Diese Entfremdung gilt es, so Debra Kelly, für die Autoren ebenso wie für ihre Protagonisten durch einen «intellectual effort» ${ }^{61}$ zu überwinden.

Die Schule macht die Protagonisten $\mathrm{zu}$ «monstre[s]» ${ }^{62}$ in den Worten Camus'. Memmis Protagonist fühlt sich «en perpétuelle rupture [...] je n'aurai jamais la solution de mon problème, car je suis problème» ${ }^{63}$. Mouloud Feraouns Protagonist bildet in dieser Hinsicht eine Ausnahme: er kehrt nach der École normale in die Kabylei zurück, auch während seiner Zeit in Algier fühlt er sich weiterhin tief verwurzelt, «[i]l sentait qu'il ne serait à l'aise que chez lui, avec des gens comme lui» ${ }^{64}$. Wohl aufgrund des kulturell homogenen dörflichen Umfelds, aus dem er stammt, gelingt es Fouroulou trotz der Prägung durch die französische Schule, seine kulturelle Identität zu bewahren. Die geistige Distanz jedoch, die Alexandre und Jacques durch ihre stetig wachsende Bildung zu ihren Familien verspüren, wiegt weit schwerer als die räumliche während Fouroulous Zeit an der École normale ${ }^{65}$. «Monster〉 oder $<$ Probleme sind Alexandre und Jacques, weil die französische Schule, nachdem sie ihnen erfolgreich vor Augen geführt hat, wie weit sie sich bereits von ihren Herkunftskontexten entfernt haben, ihnen keinen anderen Platz in der Gesellschaft anzubieten vermag. Zwar suggerieren die republikanischen Prinzipien im Falle von Jacques, dass er sich als Franzose wie jeder andere fühlen darf,

Lehrer geschrieben hat und aus dem - ungeachtet der Entwurzelung - seine große Dankbarkeit für den Lehrer hervorgeht.

61 Kelly 2005 (Anm. 34), 335.

${ }^{62}$ Camus 1994 (Anm. 3), 25, Anm. a, 284 sowie 300: «Je vais raconter l'histoire d'un monstre».

${ }^{63}$ Memmi 1966 (Anm. 29), 362.

${ }^{64}$ Feraoun 1972 (Anm. 27), 112.

${ }^{65}$ Im Falle Feraouns ist der Einfluss der École normale nicht zu unterschätzen, der, wie Fanny Colonna nachgewiesen hat, nicht allein in der Ausbildung von Lehrern bestand, sondern darauf abzielte, sie zu Mittlern zu erziehen, die assimiliert genug waren, aber Distanz zu den <indigènes` aufgebaut hatten, ohne sich als Franzosen zu fühlen: «In fact, not enough acculturation, like too much acculturation, was defined not in relation to excellence but in relation to the legitimate culture that then constituted a second center, indeed the actual center of the semantic field. What characterized all individuals located in the defect hemisphere was that they were too far from the legitimate culture. Conversely, those in the excess hemisphere were too close to it. Consequently, excellence was defined primarily in terms of distance, that is, as the sum of the images and behaviors adopted when one was neither too far nor too close to Western culture.» (Fanny Colonna: «Educating Conformity in French Colonial Algeria». In: Cooper/Stoler 1997 (Anm. 19), 346-370, hier 364). Siehe hierzu auch Fanny Colonna: Instituteurs algériens 1883-1939. Paris: Presses de la fondation nationale des sciences politiques 1975 sowie dies.: «Le système d'enseignement de l'Algérie coloniale». In: Archives Européennes de Sociologie 13 (1972), 195-220. 
seine Selbstbeschreibung als «monstre» verdeutlicht aber, dass der Protagonist sich seiner Andersartigkeit stets bewusst geblieben ist - ungeachtet seiner (im Text nicht näher spezifizierten) Berühmtheit, fühlt er sich abgelehnt: «Ce qu'ils n'aimaient pas en lui, c'était l'Algérien " ${ }^{66}$. Memmis jüdischer Protagonist muss in der Okkupationszeit erleben, wie ihm und den anderen tunesischen Juden die Bürgerrechte aberkannt werden, wie sich also das Land, das sich ihm als neue kulturelle Heimat angeboten zu haben schien, von ihm abwendet.

Die französische Schule und ihre Schulbücher vermitteln den Schülern geographische, historische, literarische und andere Kenntnisse über ein Land, das sie noch nie gesehen haben und dessen Lebensrealität mit der ihren nichts gemein hat. Die Schüler lernen, wie Albert Camus betont, ein ihnen völlig fremdes Land kennen, dessen «récits pour eux mythiques [...] étaient l'exotisme même» ${ }^{67}$. Ihre eigene Welt fehlt im Gegenzug auf den Lehrplänen, in der Bildungshierarchie dürfen die Kolonien keinen eigenen Platz beanspruchen.

Den Protagonisten wird damit die Wertlosigkeit ihres eigenen Ursprungs wie auch ihrer Umgebung plastisch vor Augen geführt, ihre Erziehung erfolgt im Hinblick auf einen Kontext, der sich ihnen - wenn überhaupt - so allenfalls später erschließen wird. Nicht nur die <indigènes, von denen einige wie Fouroulou immerhin über Väter verfügen, die Frankreich als Arbeitsmigranten kennenlernen, auch viele Algerienfranzosen waren noch nie in Frankreich. Diejenigen Differenzierungen, die im metropolitanen Frankreich aus historischen Gründen relevant sind, werden durch die Schulbücher mittransportiert und installieren in der Kolonie ein analoges System, das zudem den Interessen des Mutterlandes dient.

Die kulturellen Werte Frankreichs, die sowohl den «citoyens) als auch den 〈indigènes〉 in der Schule zugänglich gemacht werden, sollen ersteren das Bewusstsein über ihre wahre geistige wie reale Heimat - das metropolitane Frankreich - vermitteln. Dieses Bemühen, so zeigt Camus in Le premier homme, misslingt bei seinem Protagonisten Jacques ebenso wie bei seinen literarischen Vorläufern Fouroulou und Alexandre. Nicht eine Abstraktion wie die Nationalität entscheidet über das Gefühl der Zugehörigkeit, vielmehr sind es das konkrete Land und seine Traditionen, deren Präsenz jenseits der Schulbücher hier ausschlaggebend sind. Während Fouroulou seine Verbundenheit mit der Kabylei als Selbstverständlichkeit ansieht, muss Memmis Alexandre widerwillig anerkennen, dass der Orient in ihm stärkere Gefühle zu evozieren weiß als die westliche Kultur, der er so gerne völlig angehören möchte:

Après quinze ans de culture occidentale, dix ans de refus conscient de l'Afrique, peut-être faut-il que j'accepte cette évidence: ses vieilles mesures monocordes me bouleversent davantage que les grandes musiques de l'Europe. [...] Ah! Je suis

66 Camus 1994 (Anm. 3), 318.

67 Camus 1994 (Anm. 3), 137. 
irrémédiablement un barbare! ${ }^{68}$

Für Camus' Protagonisten ist Algerien seine «vraie patrie» ${ }^{69}$, außerhalb derer er sich als «monstre» fühlt. Indem Camus diese Entwicklung seines Protagonisten analog zu seinen literarischen Vorläufern anlegt, verdeutlicht er, dass die «colonial machine» ${ }^{70}$, deren Teil die Schule ist, es ungeachtet aller Bemühungen nicht vermochte, eine konsequente Trennung von «citoyens» und «indigènes» in Algerien aufrechtzuerhalten. Wichtiger als die abstrakte Tatsache der französischen Staatsbürgerschaft, die sie einem Land zuschlug, das sie nicht kannten, ist für ihn als Algerienfranzosen die unhintergehbare Präsenz des Landes, dem sie seit Generationen verbunden sind.

Die Annahme Debra Kellys, dass sich in (post)kolonialen Autobiographien gleichsam die Geburt der «independent subjectivity» ${ }^{71}$ ihrer Protagonisten manifestiere, bestätigt sich in den Texten Memmis und Camus' allenfalls als negativer Befund. Hierauf soll am Schluss noch einmal zurückgekommen werden.

\section{Allegorien für die neuen Nationen}

Die Abwesenheit Algeriens oder auch Tunesiens in den Schulbüchern, der Geschichte und der Literatur macht sie zu Orten, an denen, wie in den Herkunftsfamilien der Protagonisten, die Kultur keinen Platz hat und die damit in einer schulisch erworbenen Hierarchie von mündlicher und schriftlicher Kultur weit unter Frankreich stehen. ${ }^{72}$ Die Emanzipation der Protagonisten von diesem Bild hängt in allen drei Texten eng mit dem Bild zusammen, das von den jeweiligen Familien gezeichnet wird. An dieser Stelle möchte ich daher auf die oben bereits kurz entwickelte These zurückkommen, dass insbesondere die frühen (post)kolonialen Autobiographien Nationalallegorien entwerfen, um der im Entstehen begriffenen Identität ihres Landes literarisch Ausdruck zu verleihen. Alle drei Autoren illustrieren den Gegensatz zwischen dem über die Schule präsenten Frankreich und dem dort hartnäckig absenten Algerien bzw. Tunesien anhand der Eltern. Im Folgenden möchte ich das an der Familie und der Figur des Jacques Cormery exemplarisch vorführen, mit kurzen Verweisen auf

${ }_{68}$ Memmi 1966 (Anm. 29), 184.

${ }^{69}$ Camus 1994 (Anm. 3), 182.

70 James McClellan/François Regourd: «The Colonial Machine: French Science and Colonization in the Ancien Régime». In: Roy MacLeod (Hg.): Nature and Empire. Science and the Colonial Enterprise. Chicago: University of Chicago Press 2001, 31-50.

71 Kelly 2005 (Anm. 34), 23.

72 In der Eingangsszene des Romans Outremer von Morgan Sportès versucht ein kleiner pied noir verzweifelt, Algier auf der Frankreichkarte, die seine Mutter über seinem Bett angebracht hat, zu lokalisieren. Auch er konstatiert: «Nous savions tout sur la Seine-Saint-Denis. Rien sur l'Ouarsenis ou le djebel Amour.» Morgan Sportès: Outremer. Paris: Grasset 1989, 13. 
die Parallelen zu den beiden anderen Texten.

Der im ersten Weltkrieg gefallene, ihm folglich unbekannte und ferne Vater, der immer eine Abstraktion bleiben wird, repräsentiert Frankreich, das zwar ein Ort der (Bildungs- und Aufstiegs)Sehnsucht sein kann, zugleich aber auch essentiell unerreichbar bleiben wird. Die sorgfältig beschriftete Grabplatte des Vaters in Saint-Brieuc steht für eine okzidentale, schriftliche Erinnerungskultur, in der die Toten gezählt und benannt werden ${ }^{73}$.

Im Gegensatz dazu steht der Friedhof von Mondovi, dem Geburtsort Albert Camus' und seines Protagonisten, auf dem Sonne und Wind die Spuren der Schrift ausgelöscht haben, wenn die Toten es denn überhaupt bis auf den Friedhof geschafft haben: «La Méditerranée séparait en moi deux univers, l'un où dans des espaces mesurés les souvenirs et les noms étaient conservés, l'autre où le vent de sable effaçait les traces des hommes sur de grands espaces.» ${ }^{74}$ Der abwesende Vater wird ersetzt durch ein sich ebenfalls immer wieder entziehendes Frankreich, das, vermittelt durch die Institution der republikanischen Schule, für eine ganze Generation von Kindern der (post)kolonialen Zeit den leeren Platz usurpiert: Fouroulous Vater geht als travailleur immigré nach Frankreich, wo er bei einem Arbeitsunfall fast ums Leben kommt; Alexandres Vater leidet an schwerem Asthma, der Macht des kolonialen Verwaltungsapparats hat er nichts entgegenzusetzen ${ }^{75}$; Jacques' Vater ist im ersten Weltkrieg gefallen, als sein Sohn erst wenige Monate alt war. ${ }^{76}$

Die paternalistische Usurpation der Vaterrolle durch eine Republik, die in den so indoktrinierten jungen Leuten mehr Instrumente als Individuen sieht, bewirkt, dass sich die Auflehnung der Protagonisten im entsprechenden Alter nicht nur auf die eigenen Väter bezieht (falls diese überhaupt anwesend sind), sondern auch auf Frankreich und die französische Kultur, von der es sich ebenfalls zu emanzipieren gilt. Anders als die konkreten Väter entzieht sich die ‘väterliche〉 Republik ihnen aber immer wieder, so dass es nicht zu einer wirklichen Auseinandersetzung kommen kann.

Die Romane von Camus' Vorläufern, insbesondere von Autoren wie Albert

73 Camus 1994 (Anm. 3), 29.

${ }^{74}$ Camus 1994 (Anm. 3), 181. Es ist bezeichnend, dass Camus in diesem Zitat von «moi» spricht, sich selbst also mit seinem Protagonisten identifiziert.

${ }^{75}$ Besonders deutlich wird das in der für das Vater-Sohn-Verhältnis zentralen Szene der Ferienkolonie, Memmi 1966 (Anm. 29), $69 \mathrm{ff}$.

76 Bis hinein in die Literatur und den Film der maghrebinischen Immigration zieht sich das Thema der oft durch die Schuld Frankreichs abwesenden oder schwachen und kranken Väter, die ihren Kindern kein Vorbild zu sein vermögen, das nicht alsbald von der französischen Kultur vom Sockel gestoßen würde: in Mehdi Charefs Le the an harem d'Archi Abmed (Paris: Mercure de France 1983 sowie in der Verfilmung von 1986) hat der Vater durch einen Arbeitsunfall den Verstand verloren; Väter und Onkel in Azouz Begags Le gône du Châaba (Paris: Seuil 1986) sind der französischen Administration nicht gewachsen; in Filmen wie La haine (F 1995), L'esquive (F 2004) etc. sind die Väter meist abwesend. 
Memmi oder Driss Chraibi, sind, ganz in diesem Sinne, von einem Gestus adoleszenter Rebellion nicht nur gegen die tatsächlichen Eltern, sondern auch gegen das paternalistische koloniale System getragen. Camus' Roman hingegen ist aus der Retroperspektive eines älteren, arrivierten Protagonisten geschrieben, für den mindestens auf den ersten Blick das Aufbegehren gegen ein diskriminierendes System nicht im Vordergrund steht, sondern die Suche nach einem Ort, an dem er akzeptiert wäre.

Camus' Konstruktion Frankreichs als Repräsentantin von Schriftkultur, Geschichte und Tradition im Gegensatz zu Algerien als Ort der Anonymität und des Vergessens ist vielfach problematisiert worden, insbesondere in Bezug auf die Anlage Algeriens als des Orts, der vor der Ankunft der ersten französischen Siedler - der «premiers hommes» - ohne Geschichte und ohne Tradition war. Übersehen werden dabei aber, so meine These, mehrere Aspekte: die Dichotomie von Frankreich bzw. dem alten Europa als Ort einer über die Schrift konservierten wie zugänglich gemachten, abrufbaren Tradition im Gegensatz zu einem Maghreb der kleinen Leute, in dem die arabische Schriftkultur eine geringe Rolle spielt, ist keine, die Camus etabliert hat, vielmehr findet er sie in den Romanen Memmis, Chraibis und Feraouns vor. Diese binäre Opposition beider Kulturen ist eine koloniale Konstruktion, die vor allem durch die französische Schule tradiert und popularisiert wird. Die hierarchische Wertung, die Verachtung der indigenen Kultur, wird so erfolgreich vermittelt, dass sie auch von den maghrebinischen Autoren erst in späteren Texten hinterfragt wird.

Wichtig erscheint mir darüber hinaus ein anderer Aspekt: Camus suggeriert keineswegs in gut kolonialer Manier, mit den ersten französischen Siedlern hätten auch in Algerien Tradition und Geschichte Einzug gehalten. Das Wenige, was sie an Schriftkultur mitbringen mögen - immerhin handelt es sich, so betont er, um überwiegend arme und ungebildete Leute -, verblasst angesichts der Weite und der Macht des Landes, dem sie nun ausgeliefert sind. Dieses Land, dessen Atmosphäre und Schönheit für Camus im Zentrum vieler Texte stehen, verleibt sie sich buchstäblich ein, dezimiert sie und lässt diejenigen von ihnen, denen es gelingt zu überleben, zu einem integralen Bestandteil seiner selbst werden. Nicht ein kolonialer Gestus der Landnahme ist hierin zu sehen, sondern vielmehr sein genaues Gegenteil: Die Siedler verfallen in durchaus auch positiver Weise der schroffen Schönheit Algeriens, die auch die letzten Spuren der Schriftkultur - die Namen auf den Grabsteinen - auslöscht.

Der berechtigte Einwand, in dieser Konstruktion der Kolonie als einem Land ohne Tradition liege koloniale Herablassung, wird in meinen Augen durch andere Metaphern gekreuzt, die die zunächst eindeutig erscheinende Dichotomie von Frankreich als Land der Kultur und Algerien als Land ohne Tradition konterkariert: So entwickelt die erste Szene des Romans Algerien als neues gelobtes Land und die Geburt des Protagonisten als Nativité, ein Bild, 
dem man auf den ersten Blick sowohl Naivität als auch Hybris vorwerfen könnte, wird das Alter ego des Autors doch hier als Jesuskind geboren. Bei genauerer Betrachtung sind die Abweichungen von der biblischen Geschichte allerdings weit aussagekräftiger als die Parallelen: Das Paar armer Siedler, das bei schlechtem Wetter an seinem zukünftigen Einsatzort in Mondovi ankommt, erwartet sein erstes Kind, die Wehen haben bei Erreichen des Hauses bereits eingesetzt. Im Gegensatz zur Weihnachtsgeschichte wird ihnen aber nicht nur die Herberge nicht verwehrt, vielmehr haben sie ein eigenes Haus in diesem Dorf, das auf sie wartet. Nicht nur Kaddour, der arabische Kutscher, der sie im Pferdewagen in Bône abgeholt hat, ist sofort zur nötigen Hilfe bereit, auch die anderen Bewohner des Dorfes, Algerier wie Franzosen, öffnen bereitwillig ihre Türen und eilen hinzu, um bei der Geburt zu helfen. Mme Jacques, die Inhaberin der lokalen «cantine agricole», und die Schwiegertochter Kaddours entbinden das Kind, dem vom Vater herbeigeholten Dorfarzt bleibt nur noch, die Nabelschnur durchzuschneiden. Sieht man davon ab, dass die Schwiegertochter Kaddours ein wenig betreten darüber ist, von Henri Cormery und dem Arzt unverschleiert gesehen zu werden, ist es eine Szene großer Eintracht und Hilfsbereitschaft, in der keinerlei Unterschiede zwischen den Bevölkerungsgruppen gemacht werden.

Algerien wird in dieser Szene folglich als ein neues gelobtes Land gesehen, dass auf Neuankömmlinge nicht mit Abweisung, sondern gastfreundlich und offen reagiert. Camus etabliert es als eine Gemeinschaft der verschiedenen Bevölkerungsgruppen, die sich jenseits ethnischer oder religiöser Grenzen zusammengefunden hat. Hier, so zeigt die Eröffnungsszene, entstehen inmitten bitterster Armut neues Leben und neue Bindungen.

Frankreich ist hingegen das Land des Krieges und des Todes, der weder von der Geschichte noch von der Schriftkultur aufgehalten werden kann - im Gegenteil. Henri Cormery, Jacques’ Vater, wird zunächst 1905 als Soldat in der ersten Marokkokrise eingesetzt; bei diesem Einsatz bleibt er zumindest in ihm vertrautem maghrebinischem Territorium. Im ersten Weltkrieg dann wird er, wie viele junge Männer aus Algerien, die Frankreich nie zuvor gesehen haben, für einen Krieg eingezogen, der ihm fremd und unverständlich bleibt. Das Schiff bringt ihn auf den Weg "pour la France qu'il n'avait jamais vue, sur la mer qui ne l'avait jamais porté» ${ }^{77}$, bringt ihn «sur cette terre où il était passé fugitivement, comme un inconnu ${ }^{78}$, um schließlich dort zu sterben. Frankreich nimmt den Kindern die Väter, usurpiert ihre Position und entwurzelt die Söhne, macht aus ihnen «monstres» - von einem positiven Bild zu sprechen, wäre übertrieben.

Die Mütter sind in allen drei hier behandelten Texten - und auch da ließe die

77 Camus 1994 (Anm. 3), 67.

78 Camus 1994 (Anm. 3), 31. 
Liste sich bis weit in die Migrationsliteraturen hinein fortsetzen - dadurch charakterisiert, dass sie kaum Zugang zu Bildung haben, im Fall Camus' ist die Mutter Jacques Cormerys (wie die des Autors selbst) schwerhörig und nahezu stumm. Alle drei Mütter sind Analphabetinnen und somit von der Schriftkultur, zumal der westlichen, abgeschnitten. Ihr Verständnis für das, was ihre Söhne durch die französische Schule vermittelt bekommen, ist, wie weiter oben erläutert, gering. Für die Söhne bleiben sie gleichwohl klare affektive Bezugspunkte, die jedoch ambivalent besetzt sind: So stellt Memmis Alexandre in sich zwar Widerwillen gegen die einen berberischen Tanz vollführende Mutter fest («Elle m'était étrangère, ma mère, étrange partie de moi-même, plongée au sein des continents primitifs» ${ }^{79}$ ), muss sich aber zugleich eingestehen, dass die Musik, zu der sie tanzt, «ses vieilles mesures monocordes», «me bouleversent davantage que les grandes musiques de l'Europe ${ }^{80}$. Auch Jacques Cormery wird seiner Mutter durch die Schule entfremdet, von ihr kehrt er zurück «avec ce regard qu'il posait sur elle comme sur une étrangère» ${ }^{81}$. Die Trennung von seiner Mutter wird als schmerzlich empfunden, sie bleibt innerhalb wie außerhalb des Textes eine starke affektive Bezugsperson, die sich ihm allerdings immer entziehen wird. In einer an die Mutter gerichteten «confession» aus dem Anhang des Textes richtet der Protagonist sich an sie, um ihre Verzeihung zu erbitten: «Toi seule peut le faire [me pardonner], mais tu ne me comprends pas et ne peux me lire. Aussi je te parle, je t'écris, à toi, à toi seule, et, quand ce sera fini, je te demanderai pardon sans autre explication et tu me souriras... ${ }^{82} \mathrm{Die}$ selbst in ihrer physischen Präsenz abwesende Mutter und der allenfalls durch die französische Schule substituierte Vater bieten auf keiner Seite einen Halt, der die Herausbildung einer eigenständigen Identität vereinfachen würde. In der Abwesenheit geeigneter Vorbilder auf beiden Seiten müssen die Protagonisten sich vielmehr in der fast völligen Unabhängigkeit von ihren Eltern neu erfinden, wobei der Ablehnung und Abwesenheit der Väter, die zwar die Schriftkultur repräsentieren, aber gerade durch sie vom Tod gekennzeichnet sind, die affektive, aber distanzierte Bindung an ein Land entgegensteht, dem sie sentimental verbunden sind, von dem die Schule sie aber entfremdet hat.

Wo die Lektüre von Memmis Erstling als nationale Allegorie naheliegend erscheint, mag sie für Camus' letztes Werk zunächst überraschend sein. Der Entwurf seines Protagonisten nach dem Bild des neuen Algeriens, das Camus sich vorstellt, zeigt aber, dass er sich der Probleme, die die Konstruktion eines solch neuen Zusammenlebens zwischen den verschiedenen Gruppen mit sich bringen würde, bewusst war, ohne dafür einfache Lösungen anbieten zu kön-

\footnotetext{
79 Memmi 1966 (Anm. 29), 181.

80 Memmi 1966 (Anm. 29), 184.

81 Camus 1994 (Anm. 3), 229.

82 Camus 1994 (Anm. 3), 319.
} 
nen. Der Blick, den er unter dem Mantel der Allegorie auf das väterliche Frankreich wirft, ist düster, der auf das mütterliche Algerien mit vielen offenen Fragen versehen.

Debra Kelly geht davon aus, dass die von ihr untersuchten postkolonialen Autobiographien letztlich Lösungen anzubieten vermögen:

These writers [...] are also all clear on the need to write themselves out of dominant systems of representation, to remove themselves as inventions of the colonial imagination, while simultaneously decolonising themselves from that colonial imagination and from their own ambivalent desire for the West; to posit themselves as subjects. ${ }^{83}$

In meinen Augen bieten die von Kelly untersuchten Texte wie auch der von mir in denselben Kontext gestellte Roman von Camus keinen Ausweg.

Stellvertretend für die sich herausbildenden, nach Unabhängigkeit strebenden Nationen, sind die Protagonisten sich der jenseits der rationalen Ebene unabweisbaren, starken emotionalen Verbundenheit mit dem Maghreb selbst ebenso bewusst wie ihrer Ablehnung eines paternalistischen französischen Staats, der sie ihrerseits ablehnt. Der Wunsch nach einer individuellen und letztlich auch nationalen Identität jenseits der französischen ist die Folge dieser Abweisung durch Frankreich und entsteht in der Logik der Texte von Camus und Memmi aus dem Bedürfnis nach einem (dritten) Ort, an dem man bedingungslos akzeptiert wäre. Dass dieser Ort aber auch mit dem Algerien oder Tunesien jenseits des kolonialen Apparats nicht identisch sein kann, verdeutlichen die Texte. Die unabgeschlossene Suche nach einer bislang nur imagined community», in der sie weder «monstre(s)» noch «problème(s)» sind, ist daher vielleicht mehr als im doppelten Sinne intellektueller Entwurf zu verstehen denn als Modell eines jungen Nationalstaats. Die Romane können die Andersartigkeit der Individuen wie der sich herausbildenden Nationen nicht harmonisch aufheben. Ebenso wenig wie es den Texten gelingt, für ihre Protagonisten Kohärenz und Einheit zu schaffen, gelingt es, die Länder bei ihrer Unabhängigkeit als kohärente Einheiten zu etablieren. Die Schwierigkeit dieses Prozesses zwischen Loslösung und Annäherung wird sowohl bei Memmi als auch bei Camus deutlich.

Camus' Entscheidung, diesen Text, den er als seinen bis dahin wichtigsten ansah, literarischen Vorbildern anzuverwandeln, die ganz dezidiert nicht aus Frankreich, sondern aus dem Maghreb kamen und im Zeichen der Unabhängigkeitsbewegungen der dortigen Kolonien stehen, kann als Abwendung von dem Land verstanden werden, das ihn berühmt, aber auch zum «Monster gemacht hat. Die literarische Hinwendung zu den Autoren, die in ihren Texten die Probleme der jungen postkolonialen Nationen präfigurieren, bedeutet

\footnotetext{
${ }^{83}$ Kelly 2005 (Anm. 34), 335.
} 
ebenso wenig die emphatische Bejahung einer algerischen Unabhängigkeit im Sinne der FLN. Camus entscheidet sich, sein Schweigen zugunsten einer Alternative zu brechen, mit der er hofft, allen in Algerien verwurzelten Bevölkerungsgruppen gerecht zu werden. 